\title{
Crisis epilépticas generalizadas asociadas a intoxicación pasiva por cocaína en un lactante
}

\author{
Generalized epileptic seizures in an infant due to passive exposure to \\ cocaine
}

\author{
S. Aguilera' ${ }^{1}$, C. Salado² , I. Díaz López ${ }^{2}$, J.I. Montiano², M.P. Botella ${ }^{2}$
}

\section{RESUMEN}

Fundamento. Las crisis convulsivas sin fiebre en lactantes implican un diagnóstico diferencial amplio.

\begin{abstract}
Caso clínico. Lactante mujer de 7 meses que presenta, estando afebril, dos crisis generalizadas tónico-clónicas breves en 23 horas. Su desarrollo psicomotor y estaturo-ponderal seguía un curso normal. Se obtuvo analítica de sangre, ecografía cerebral y electroencefalograma, normales. Debido a una situación sociofamiliar desfavorable, se realizaron tóxicos en orina, positivos para cocaína en dos muestras sucesivas; se negativizaron en 48 horas. Estuvo en contacto con humo ambiental horas previas al inicio de las crisis. Sin crisis en los siguientes 6 meses, con desarrollo normal, sigue bajo vigilancia por asistencia social.
\end{abstract}

Discusión. La inhalación pasiva de humo de cocaína se asocia a crisis convulsivas en lactantes. Este riesgo es casi desconocido en nuestro país, a pesar del aumento del consumo de crack. Ante una primera crisis epiléptica afebril en cualquier edad, se debe incluir de forma rutinaria la detección de tóxicos en orina.

Palabras clave. Cocaína. Crack. Crisis epiléptica. Exposición pasiva. Intoxicación.

\begin{abstract}
Background. The differential diagnosis of afebrile seizures in the first year of life is extensive.

Case report. A 7-month old infant presented two afebrile generalized tonic-clonic seizures in 23 hours; her psychomotor and growth development followed a normal course. Laboratory analysis, cerebral echography and electroencephalogram were normal. Urine toxicology was positive for cocaine on two occasions. A negative urine sample was obtained 48 hours later. The parents denied drug abuse but explained a recent exposure to smoke some hours before the episode. The patient had no seizures in the following six months, with normal psychomotor development.
\end{abstract}

Discussion. Passive inhalation of cocaine is associated with seizures in infants. There is no perception of the risk of passive exposure to cocaine in our country, despite the increasing consumption of crack in young adults. Urine toxicology should be systematically included in the study of a first afebrile seizure in an infant.

Key words. Cocaine. Crack. Epileptic seizure. Intoxication. Passive smoking.
1. Servicio de Pediatría. Unidad de Neuropediatría. Hospital Virgen del Camino. Servicio Navarro de Salud. Pamplona.

2. Servicio de Pediatría. Servicio Vasco de SaludOsakidetza. Hospital de Txagorritxu. Vitoria.

\section{Correspondencia:}

Sergio Aguilera

Servicio de Pediatría

Hospital Virgen del Camino

31008 Pamplona 


\section{INTRODUCCIÓN}

La cocaína produce numerosos efectos adversos sobre el sistema nervioso central. Su consumo en adultos y adolescentes puede producir accidentes cerebrovasculares, crisis epilépticas, cefalea de tipo migrañoso y alteraciones extrapiramidales y del estado mental ${ }^{1,2}$. La exposición prenatal a cocaína se ha relacionado en el recién nacido con una mayor frecuencia de sufrimiento fetal agudo, prematuridad, bajo peso y hemorragia subependimaria ${ }^{3}$; y en el niño mayor con retraso del crecimiento, dificultades de atención sostenida y necesidades educativas especiales ${ }^{4-6}$. En lactantes y niños menores de 8 años las crisis convulsivas son la manifestación neurológica aislada más frecuente de la intoxicación por cocaína, que ocurre en la mayoría de los casos mediante exposición pasiva, por lo que supone un riesgo potencial para la salud de los niños que conviven con consumidores habituales o esporádicos ${ }^{7,8}$. Presentamos el caso de una lactante de 7 meses con dos crisis convulsivas afebriles en la que se detectó cocaína en orina, probablemente relacionada con exposición pasiva a humo que contenía esta sustancia.

\section{CASO CLÍNICO}

Lactante de sexo femenino que ingresó a los 7 meses de vida por presentar una crisis generalizada tónico-clónica breve, sin proceso infeccioso ni fiebre asociada, que se repitió con similares características a las 23 horas del primer episodio, estando ingresada. No se observó inicio focal de la crisis, con constantes vitales mantenidas, exploración física y neurológica posterior normal. Los padres no referían antecedentes personales a destacar, acudía a las revisiones periódicas de salud y su desarrollo psicomotor y estaturo-ponderal seguía un curso normal. A su ingreso se realizó hemograma, pruebas de función renal, hepática, reactantes de fase aguda, ecografía cerebral y electroencefalograma, con resultado normal. Ante la observación de una situación sociofamiliar conflictiva, se realizó la búsqueda de tóxicos en orina, que resultaron positivos para cocaína en dos muestras sucesivas. La primera muestra arrojó un nivel cuantificado de metabolitos de cocaína en orina de $1,7 \mu \mathrm{g} / \mathrm{ml}$ y la segunda, recogida pocas horas después, 0,4 $\mu \mathrm{g} / \mathrm{ml}$ (punto de corte de nuestro laboratorio en $0,3 \mu \mathrm{g} / \mathrm{ml}$ ), que se negativizaron a las 48 horas del ingreso. El padre es fumador habitual de cannabis, pero los familiares negaron el consumo de cocaína en su domicilio ni en su ambiente sociofamiliar cercano y apuntaron la posibilidad de contacto con humo ambiental en un local cerrado donde estuvieron horas antes del inicio de las crisis. Bajo vigilancia por la asistente social asignada desde el alta, la paciente no ha vuelto a presentar crisis en los siguientes seis meses y el desarrollo psicomotor sigue un curso normal.

\section{DISCUSIÓN}

En este caso, el tipo de exposición pasiva a la cocaína más probable fue la de inhalación de humo ambiental, descrito en la literatura como causa más frecuente en este grupo etario ${ }^{7,8}$. Dada la ausencia de otros síntomas neurológicos y la rápida recuperación clínica de la niña, la ingestión accidental fue menos probable y, por el seguimiento posterior sin incidencias, no parece probable tampoco la intoxicación voluntaria por los cuidadores.

La acción estimulante de la cocaína sobre el sistema nervioso central se debe al bloqueo de la recaptación presináptica de catecolaminas y serotonina, provocando un exceso de neurotransmisores en la hendidura sináptica. La activación del sistema nervioso simpático produce una vasoconstricción que predispone a la aparición de crisis epilépticas, sobre todo en niños susceptibles $^{1,9,10}$. La vida media de la cocaína en el organismo es de aproximadamente una hora. Se metaboliza mediante la acción de las colinesterasas plasmáticas y hepáticas y se excreta por la orina, pudiendo detectarse hasta 24-48 horas después de su consumo o exposición pasiva. La disminución de la actividad de la colinesterasa plasmática en el feto y lactante los hace especialmente sensibles a la acción de esta sustancia, detectando los metabolitos de la cocaína en la orina con más frecuencia que en niños mayores y favoreciendo la aparición de síntomas de intoxicación ${ }^{9,11-13}$.

La cocaína es la segunda droga psicoactiva ilegal más consumida en España: el 8\% de la población de nuestro país entre 15-64 
años la ha probado alguna vez y un 1,6\% la ha consumido en el último mes. Aunque el consumo de cocaína en polvo está estabilizado en los últimos años, está aumentando la proporción de personas que la prueban en forma de base o crack para fumar ${ }^{14} \mathrm{o}$ impregnan con polvo el cigarrillo de tabaco. A pesar de estos datos, en España no se han realizado estudios sobre la frecuencia de la exposición pasiva a cocaína postnatal y tan sólo se han comunicado casos ais$\operatorname{lados}^{9,15}$. En EEUU se ha comprobado que en niños menores de 8 años el mecanismo de exposición más frecuente a la cocaína es la intoxicación pasiva mientras el niño está en la habitación donde se fuma o se ha fumado cocaína base ${ }^{7,8}$. Estimaron además que la incidencia de exposición pasiva oculta a la cocaína, medida en muestras de orina en niños que consultaron en urgencias hospitalarias por cualquier motivo, oscilaba entre un 2,4 y un $36 \%$, según la población estudiada y la sensibilidad del método de detección utilizado ${ }^{8,12,16}$. Estos resultados positivos se correlacionaban con la presencia de síntomas de vías respiratorias inferiores y superiores frecuentes que motivaban las consultas a urgencias, posiblemente relacionados con la exposición pasiva de las vías aéreas al humo de cocaína y tabaco ${ }^{8}$.

Con la comunicación de este caso y ante el aparente aumento del consumo de cocaína base en España, es necesario insistir en la importancia de realizar detección de tóxicos en orina en todo niño que acuda a urgencias con una crisis epiléptica afebril sin diagnóstico etiológico previo. La evolución clínica suele ser satisfactoria, pero la vigilancia y apoyo social deben ser parte del tratamiento en estos casos para evitar recurrencias y complicaciones mayores. Otros autores amplían aún más esta recomendación, incluyendo crisis de inicio focal o asociadas a fiebre, descritas también en niños entre 12 meses y 8 años de edad con exposición pasiva a humo de cocaína ${ }^{7}$. Si durante el seguimiento las crisis convulsivas se repitieran en presencia de cocaína en orina, habría que descartar la exposición crónica mediante determinación de la sustancia y sus metabolitos en un análisis segmentario del pelo ${ }^{11,15}$. Por último, es importante tener en cuenta la baja percepción de riesgo de los cuidadores que fuman tabaco o cocaína base en la misma habitación que el niño o le exponen a ambientes de humo y fomentar campañas informativas sobre los riesgos de exposición pasiva a drogas en lactantes y niños.

\section{BIBLIOGRAFÍA}

1. Blanco M, Díez-Tejedor E, Vivancos F, Barreiro P. Cocaína y enfermedad cerebrovascular en adultos jóvenes. Rev Neurol 1999; 29: 796800.

2. Martínez-Vila E, Irimia P. Factores de riesgo del ictus. An Sist Sanit Navar 2000; 23 (Suppl 3): 25-31.

3. Mur Sierra A, García-Algar O, López-Segura N. Toxicidad de la cocaína en el recién nacido. Detección y prevalencia. Identificación de factores de susceptibilidad. An Esp Pediatr 2002; 56: 241-246.

4. Richardson GA, GoldSChMidT L, LARKBy C. Effects of prenatal cocaine exposure on growth: a longitudinal analysis. Pediatrics 2007; 120: e1017-1027.

5. Bandstra ES, Morrow CE, Anthony JC, AccorNERO VH, FRIED PA. Longitudinal investigation of task persistence and sustained attention in children with prenatal cocaine exposure. Neurotoxicol Teratol 2001; 23: 545-559.

6. Levine TP, Liu J, Das A, Lester B, Lagasse L, SHANKARAN $S$ et al. Effects of prenatal cocaine exposure on special education in schoolaged children. Pediatrics 2008; 122: e83-91.

7. MotT SH, PACKER RJ, Soldin SJ. Neurologic manifestation of cocaine exposure in childhood. Pediatrics 1994; 93: 557-560.

8. Lustbader AS, Mayes LC, McGee BA, Jatlow P, ROBERTS WL. Incidence of passive exposure to crack/cocaine and clinical findings in infants seen in an outpatient service. Pediatrics 1998; 102: e5.

9. García Peñas JJ, Ramos Lizana J, Martínez-Bermejo A, Díaz González C, Pascual Castroviejo I. Acute cocaine poisoning in an infant. An Esp Pediatr 1990; 33: 582-583.

10. Fernández-EsPejo E. Neurobiología de la adicción a psicoestimulantes. Rev Neurol 2006; 43: 147-154.

11. Garcia-Bournissen F, Nesterenko M, Karaskov T, Koren G. Passive environmental exposure to cocaine in Canadian children. Paediatr Drugs 2009; 11: 30-32. 
12. Kharasch SJ, Glotzer D, Vinci R, Weitzman M, SARGENT J. Unsuspected cocaine exposure in young children. Am J Dis Child 1991; 145: 204-206.

13. Gainza I, Nogué S, Martínez Velasco C, Hoffman RS, Burillo-Putze G, Dueñas A et al. Intoxicación por drogas. An Sist Sanit Navar 2003; 26 (Suppl. 1): 99-128.

14. Ministerio de SANidAd y Consumo. Delegación del gobierno para el plan nacional sobre drogas. Informe de la encuesta domiciliaria sobre alcohol y drogas en España 2007/08. Disponible en: http://www.pnsd.msc.es/Categoria2/ observa/pdf/InformeEdades2007-2008.pdf

15. García-Algar O, LóPez-Vílchez MA, Pacifici R, Pichini S. Intoxicación aguda y exposición crónica a cocaína en un niño. Med Clin (Barc) 2005; 125: 436-439.

16. Rosenberg NM, Meert KL, Marino D, Yee H, KaUFFMAN RE. Occult cocaine and opiate exposure in children and associated physical findings. Pediatr Emerg Care 1995; 11: 167-169. 